Article

\title{
Do Transitive Preferences Always Result in Indifferent Divisions?
}

\author{
Marcin Makowski ${ }^{1,}$, Edward W. Piotrowski ${ }^{1}$ and Jan Sładkowski ${ }^{2}$ \\ ${ }^{1}$ Institute of Mathematics, University of Bialystok, Ciołkowskiego 1M, Pl-15245 Białystok, Poland; \\ E-Mail: qmgames@gmail.com \\ ${ }^{2}$ Institute of Physics, University of Silesia, Uniwersytecka 4, Pl-40007 Katowice, Poland; \\ E-Mail: jan.sladkowski@us.edu.pl \\ * Author to whom correspondence should be addressed; E-Mail: makowski.m@gmail.com; \\ Tel.: +48-85-738-8279; Fax: +48-85-738-8300.
}

Academic Editor: Demosthenes Ellinas, Giorgio Kaniadakis, Jiannis Pachos and Antonio M. Scarfone

Received: 9 January 2015 / Accepted: 17 February 2015 / Published: 2 March 2015

\begin{abstract}
The transitivity of preferences is one of the basic assumptions used in the theory of games and decisions. It is often equated with the rationality of choice and is considered useful in building rankings. Intransitive preferences are considered paradoxical and undesirable. This problem is discussed by many social and natural scientists. A simple model of a sequential game in which two players choose one of the two elements in each iteration is discussed in this paper. The players make their decisions in different contexts defined by the rules of the game. It appears that the optimal strategy of one of the players can only be intransitive (the so-called relevant intransitive strategy)! On the other hand, the optimal strategy for the second player can be either transitive or intransitive. A quantum model of the game using pure one-qubit strategies is considered. In this model, an increase in the importance of intransitive strategies is observed: there is a certain course of the game where intransitive strategies are the only optimal strategies for both players. The study of decision-making models using quantum information theory tools may shed some new light on the understanding of mechanisms that drive the formation of types of preferences.
\end{abstract}

Keywords: relevant intransitive strategies; quantum strategy; quantum modeling; decision-making 


\section{Introduction}

Games have always fascinated scholars, often contributing to the development of new theories [1]. In fact, the attempts to construct a systematic theory of rational behavior are focused on games as simple examples of human rationality. The attractiveness of such an approach to the analysis of the interactions between rational players (a problem reflected in many fields of science) reveals itself in its various applications [2]. Game theory methods have been used in areas, such as military science, biology, economics and other social sciences. Since the very beginning, game theory has been closely connected to information theory [3]. Therefore, during the development of the theory of quantum information [4], a quantum game theory has emerged in a natural way. In its general form, pure strategies are identified with Hilbert space vectors (pure states)—qubits or qubit systems. Mixed strategies are represented by convex combinations of pure states. Replacing classical probabilities used in game theory with quantum probability amplitudes provides many interesting opportunities arising from superposition and entanglement. The idea of constructing quantum models of games is intensely developing nowadays. Numerous examples show the rapid rate with which the situation of players changes after obtaining access to quantum technology and how the fundamental limitations of classical models can be overcome thanks to the properties of quantum processes [5-9].

This paper considers a simple model of a repeated game in which, at each stage, both players divide between themselves a set consisting of three goods. With reference to the earlier results [10-15] influenced by the remarks of Hugo Steinhaus [16], the players are referred to as "cats" and goods are referred to as "foods". The main goal of our analysis is to examine how the rules of the game affect the type of optimal strategies for the players (whether they are transitive or intransitive). The differences arising from the adoption of a different way of sourcing strategies are also examined. One of them is a mixed strategy based on one bit (classical variant), and the other is a one-qubit pure state (quantum variant).

Before we proceed to the formal description of the game, let us highlight the main ideas of transitivity and intransitivity. This is done in the next subsection. The second subsection of this Introduction is devoted to a review of recent results that have led us to formulate the main results.

\subsection{Intransitivity}

Any relation $\succ$ between the elements of a certain set is called transitive if:

$$
A \succ B \wedge B \succ C \Rightarrow A \succ C
$$

is fulfilled for any of three elements $A, B, C$. If this condition is not fulfilled, then the relation will be called intransitive (not transitive).

The problem of transitivity (intransitivity) stems from various fields of research. There is an opinion that people who make decisions relying on rational reasoning should make decisions in determined and linear order $[17,18]$. The transitivity of preferences indicates the way of choosing according to the "logical order". There is also a hypothesis that many animals (also people) follow transitive inference rules (choosing $A$ over $C$ on the basis of knowing that $A$ is better than $B$ and $B$ is better than $C$ ). This type of reasoning has been confirmed in several animal species [19]. 
One of the main arguments against intransitiveness is the so-called "money pump" [20]. On the other hand, some modifications of utility theory and decision theory, which dispense of the transitivity assumption, have been considered [21,22].

The beginnings of research on intransitive orders probably dates back to 1785, when Jean Condorcet published his work, Essay on the Application of Analysis to the Probability of Majority Decisions (1785), in which he analyzed the paradox of voting. He concluded that collective preferences can be intransitive even if the preferences of individual voters are not. Analysis of this paradox led Kenneth Arrow (Nobel Prize winner in economy) to prove that an election procedure that would perfectly agree with the basic postulates of democracy does not exist [23].

In psychology, which also attempts to explain, inter alia, the decision making process, special interest is focused on a broadly understood relation of superiority or domination (preference) [24,25]. Does the fact that Adominates over B and B dominates over C imply that A dominates over C? It turns out that the answer is not obvious and depends on a particular situation. The discussion on the concept of dominance has also continued using the tools provided by exact sciences with probabilistic models as good examples, e.g., Efron's dice [26]. The game proposed by Walter Penney [27] is another example of intransitivity in probabilistic models. Intransitivity may explain the processes occurring in nature. Rivalry between species may be intransitive. For example, in the case of fungi, Phallus impudicus replaced Megacollybia platyphylla, M. platyphylla replaced Psathyrella hydrophila, but P. hydrophila replaced P. impudicus [28]. Similarly, we can explain the stability of the population of lizards [29] or experiments with bees, which make intransitive choices between flowers [30]. Intransitivity models appear also in many seemingly distant sciences, including philosophy [17], operations research [31], thermodynamics [32], quantum theory [12] and logic [33].

Research on the rational decision-making process is complex and consists of many various concepts. There is a large literature with discussions on rational choice [34]. This is a difficult problem, because it turns out that the assumptions made by theoreticians (such as completeness, transitivity, the independence of irrelevant alternatives or others) are often broken in the course of experiments with humans. Thus, people have constructed theories of choice without transitivity or other assumptions. In this paper, we focus on one of the most important issues in the theory of decision: the transitivity/intransitivity of the choice. Reflections on this occurs in the context of studies of various types of preferences, state-dependent preferences [35] and context-dependent preferences [36].

The issue of intransitivity is still not well understood. Therefore, attempts to understand it in the framework of quantum game theory have been made. This new approach leads to many interesting conclusions that can be used in research on decision-making process simulation and other biological mechanisms. Interesting and very promising concepts of quantum structure in cognition are currently being considered [37].

\subsection{Earlier Results: The "I Cut, You Choose" Game.}

Hugo Steinhaus mentions Pitts' experiments with cats in his diary [16]. It turns out that the cat, facing the choice between fish, meat and milk, prefers fish to meat, meat to milk and milk to fish! Steinhaus thought that the cat provided itself with a balanced diet, thanks to the above-mentioned food 
preferences. This is one of the key factors needed to maintain good health. Hence, in our model of the game, the players are cats choosing among various types of food. Obviously, this is only an illustration of the problem, which can be interpreted in different ways (e.g., in relation to other goods or as an electoral issue).

A "classical" model of a game in which the player (cat) is offered three types of foods, every time, in pairs of two types, was put forward in the paper [10]. The optimal strategy was defined as one that leads to a balanced diet (equal distribution of the frequency of the occurrence of a particular food in the cat's diet). The offering player (nature) was not interested in the result of the game. The quantitative analysis of various types (intransitive or transitive) of optimal strategies indicated the advantage of the transitive strategies. Intransitive strategies represent a significant part of all optimal strategies, but in the situation that favors optimal strategies, we can always find the strategy that determines the transitive order (with an identical result, under the same conditions). This situation changes in the quantum variant of the game [11], which reveals the existence of the so-called relevant intransitive strategies, defined as follows [14]:

Definition 1. The intransitive strategy will be called the relevant strategy, if there is no transitive strategy of the same consequences under the same assumptions.

Paper [14] considered electoral interpretation of the game (see also [38]). The decrease of the importance of intransitive orders, which accompanies the growth of the support for one of the candidates (if voters start to prefer one of the candidates), turned out to be an interesting property of the quantum game model. The use of an entangled state for the model construction causes an increase in the importance of transitive strategies.

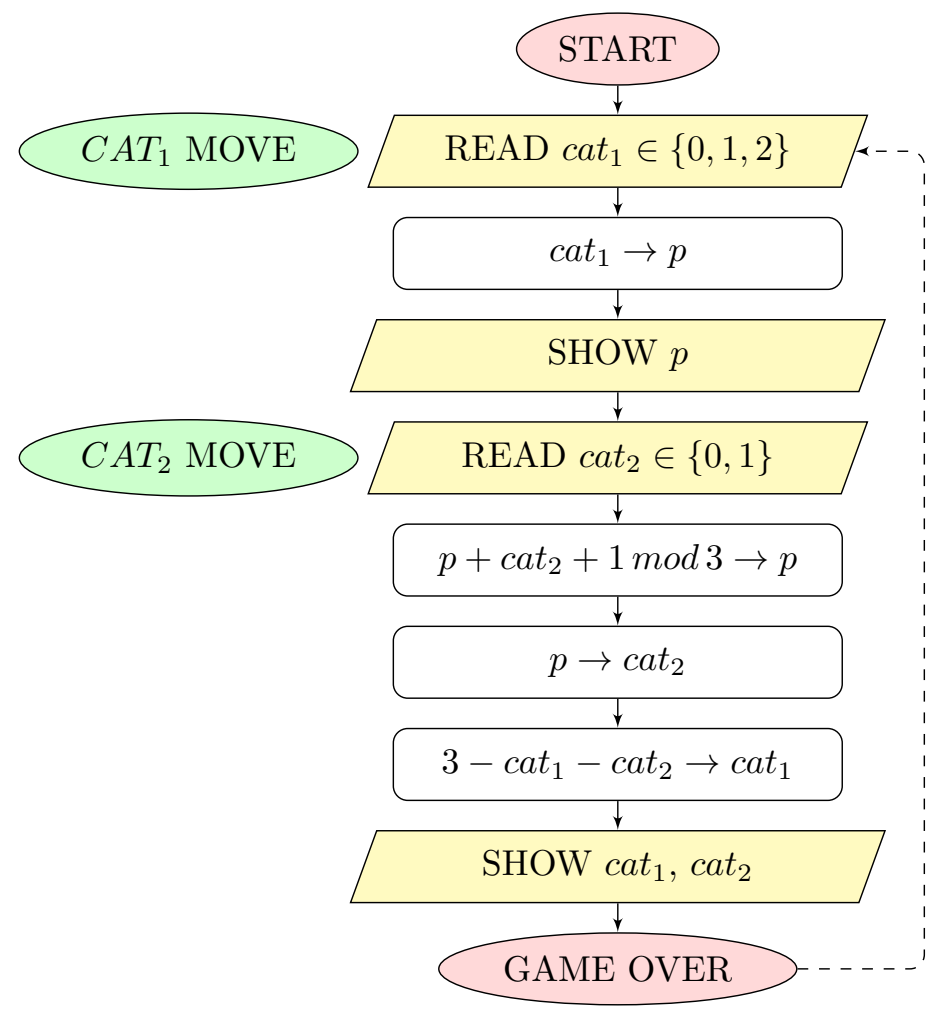

Figure 1. Flow diagram of each iteration of the "I cut, you choose" game. 
It turns out that intransitive relevant strategies are not only characteristic of quantum models. In the paper [15] the offering player (nature) has been replaced by a rational player. In this game, both players (Cat 1 and Cat 2) divide the set of three foods according to the "I cut, you choose" method [39,40] (see Figure 1). The first player chooses and rejects one of the foods. Then, the second player selects and consumes one of the remaining two foods. The first player eats the food that is left. The optimum consists (as in previous models) in not distinguishing between any of the three foods. All types of food are equally important to each of the players.

In this variant of the game, only the second player (Cat 2) makes choices between the pairs of food offered to him. Therefore, transitive and intransitive strategies can be analyzed only in the case of this player. It appears that his optimal strategies are exclusively intransitive (relevant intransitive strategies).

In this article, a modification of the above game is analyzed. It consists of letting the second player choose the food selected at the first stage of the game, as well as the food rejected by the other player. This modification allows both players to determine their preferences with regard to the pairs of food. This will enable us to explore the types of strategies and their availability for individual players.

\section{Classical Model}

The two players (Cat 1 and Cat 2) are offered three foods (Food 0, Food 1, Food 2), always in pairs of two, in accordance with the following procedure. The first player (Cat 1) selects and keeps one of the three foods. The second player (Cat 2) chooses between the two remaining foods. The food kept in the first move and the food rejected by Cat 2 constitute now a pair from which a selection is made by Cat 1 (second move). A diagram of the game is shown in Figure 2.

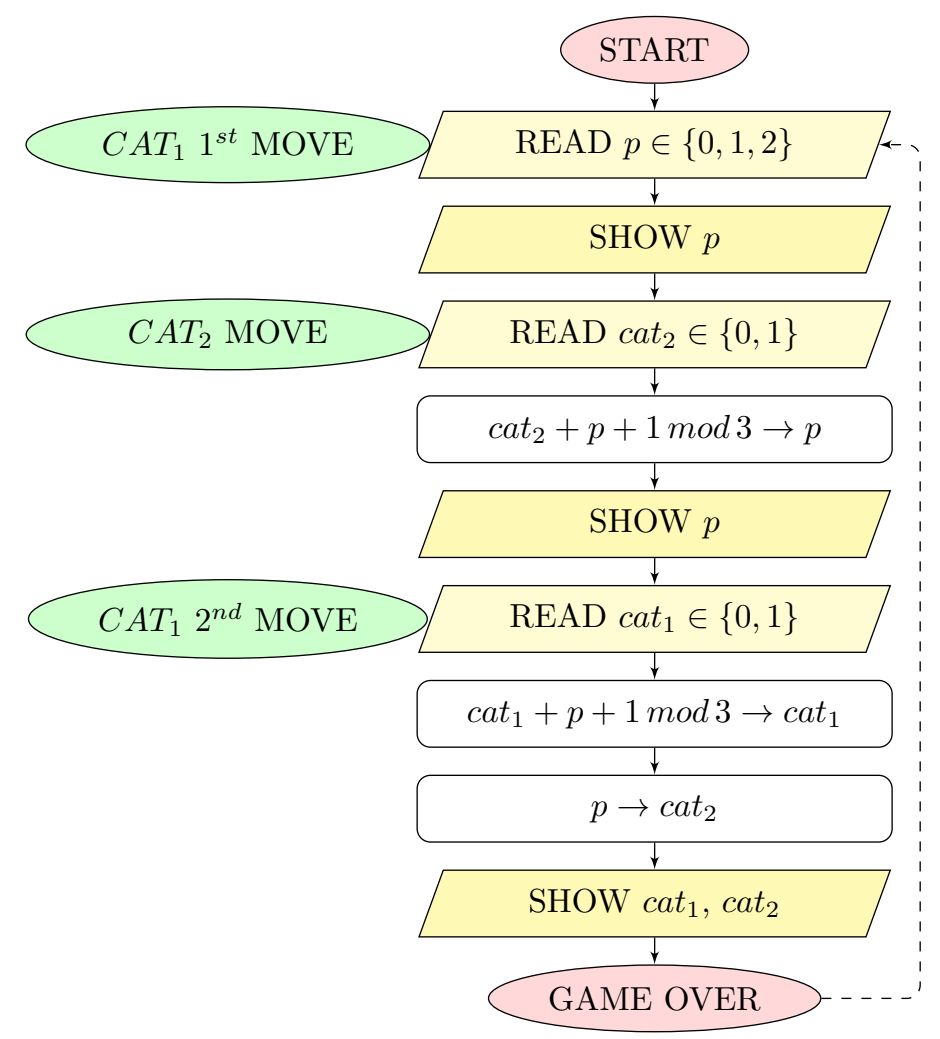

Figure 2. Flow diagram of each iteration of of the game. 
The optimal behavior of the players is not to favor any of the three foods (each being equally attractive and important). Both participants try to achieve a balanced diet. This assumption allows one to analyze the meaning of intransitive orders, because all of the elements that we sort are equally important.

\subsection{Mathematical Description}

The first move of Cat 1 is limited to selecting one of the three food. This movement can be described by the point $\left(P_{0}, P_{1}, P_{2}\right)$ of a three-dimensional simplex, where $P_{j}$ denotes the frequency of the choice of food labeled by $j$. Let us denote by $P\left(C_{k} \mid B_{j}\right)$ the probability of choosing (by Cat 2) food $k$ when the offered pair of dishes does not contain food $j$. Analogously, $Q\left(C_{k} \mid B_{j}\right)$ denotes the probability of Cat 1 choosing food $k$ when the offered pair of dishes does not contain food $j$. We refer here to the mixed strategies as pure strategies, which do not lead to optimal results. Six conditional probabilities $P\left(C_{k} \mid B_{j}\right)$ (or $Q\left(C_{k} \mid B_{j}\right)$ ) determine the behavior of the players with respect to the pairs of food portions proposed to them.

Let $\lambda_{k}$ and $\omega_{k}$ denote the frequencies of the appearance of the particular foods in Cat 1 and Cat 2's diet, respectively.

For Cat 2, we obtain:

$$
\begin{aligned}
& \omega_{0}=P\left(C_{0} \mid B_{1}\right) P_{1}+P\left(C_{0} \mid B_{2}\right) P_{2}, \\
& \omega_{1}=P\left(C_{1} \mid B_{0}\right) P_{0}+P\left(C_{1} \mid B_{2}\right) P_{2}, \\
& \omega_{2}=P\left(C_{2} \mid B_{0}\right) P_{0}+P\left(C_{2} \mid B_{1}\right) P_{1} .
\end{aligned}
$$

From Cat 1's point of view:

$$
\begin{aligned}
& \lambda_{0}=Q\left(C_{0} \mid B_{1}\right)\left[P\left(C_{1} \mid B_{0}\right) P_{0}+P\left(C_{1} \mid B_{2}\right) P_{2}\right]+Q\left(C_{0} \mid B_{2}\right)\left[P\left(C_{2} \mid B_{0}\right) P_{0}+P\left(C_{2} \mid B_{1}\right) P_{1}\right] \\
& \lambda_{1}=Q\left(C_{1} \mid B_{2}\right)\left[P\left(C_{2} \mid B_{0}\right) P_{0}+P\left(C_{2} \mid B_{1}\right) P_{1}\right]+Q\left(C_{1} \mid B_{0}\right)\left[P\left(C_{0} \mid B_{1}\right) P_{1}+P\left(C_{0} \mid B_{2}\right) P_{2}\right], \\
& \lambda_{2}=Q\left(C_{2} \mid B_{1}\right)\left[P\left(C_{1} \mid B_{0}\right) P_{0}+P\left(C_{1} \mid B_{2}\right) P_{2}\right]+Q\left(C_{2} \mid B_{0}\right)\left[P\left(C_{0} \mid B_{1}\right) P_{1}+P\left(C_{0} \mid B_{2}\right) P_{2}\right] .
\end{aligned}
$$

Let us introduce the following parameterization of the conditional probabilities:

$$
\begin{array}{ll}
P\left(C_{2} \mid B_{0}\right)=\frac{1+l_{0}}{2}, & P\left(C_{1} \mid B_{0}\right)=\frac{1-l_{0}}{2}, \\
P\left(C_{0} \mid B_{1}\right)=\frac{1+l_{1}}{2}, & P\left(C_{2} \mid B_{1}\right)=\frac{1-l_{1}}{2}, \\
P\left(C_{1} \mid B_{2}\right)=\frac{1+l_{2}}{2}, & P\left(C_{0} \mid B_{2}\right)=\frac{1-l_{2}}{2} .
\end{array}
$$

and:

$$
\begin{array}{ll}
Q\left(C_{2} \mid B_{0}\right)=\frac{1-L_{0}}{2}, & Q\left(C_{1} \mid B_{0}\right)=\frac{1+L_{0}}{2} \\
Q\left(C_{0} \mid B_{1}\right)=\frac{1-L_{1}}{2}, & Q\left(C_{2} \mid B_{1}\right)=\frac{1+L_{1}}{2} \\
Q\left(C_{1} \mid B_{2}\right)=\frac{1-L_{2}}{2}, & Q\left(C_{0} \mid B_{2}\right)=\frac{1+L_{2}}{2} .
\end{array}
$$




\subsection{Optimal Strategies}

The optimal strategy for the two players is to get an equal distribution of the frequency of the occurrence of a particular food in the diet. Therefore, the following conditions must be fulfilled:

$$
\begin{aligned}
& \lambda_{0}=\lambda_{1}=\lambda_{2}=\frac{1}{3} \\
& \omega_{0}=\omega_{1}=\omega_{2}=\frac{1}{3} .
\end{aligned}
$$

Condition (7) takes the form:

$$
\begin{aligned}
& l_{1} P_{1}-l_{2} P_{2}=\frac{2}{3}-\left(P_{1}+P_{2}\right), \\
& l_{2} P_{2}-l_{0} P_{0}=\frac{2}{3}-\left(P_{0}+P_{2}\right), \\
& l_{1} P_{1}-l_{3} P_{3}=\frac{2}{3}-\left(P_{0}+P_{3}\right),
\end{aligned}
$$

and its solution:

$$
\begin{aligned}
& P_{0}=\frac{-\left(-1-l_{1}+l_{2}-3 l_{1} l_{2}\right)}{3\left(1+l_{0} l_{1}+l_{0} l_{2}+l_{1} l_{2}\right)}, \\
& P_{1}=\frac{-\left(-1+l_{0}-l_{2}-3 l_{0} l_{2}\right)}{3\left(1+l_{0} l_{1}+l_{0} l_{2}+l_{1} l_{2}\right)}, \\
& P_{2}=\frac{-\left(-1-l_{0}+l_{1}-3 l_{0} l_{1}\right)}{3\left(1+l_{0} l_{1}+l_{0} l_{2}+l_{1} l_{2}\right)},
\end{aligned}
$$

defines a mapping of the three-dimensional cube $[-1,1]^{3}$ in the space of parameters $l_{j}$ (that define conditional probabilities $P\left(C_{k} \mid B_{j}\right)$ ) into a triangle (two-dimensional simplex). The barycentric coordinates of a point of this triangle are interpreted as the probabilities $\left(P_{0}, P_{1}, P_{2}\right)$.

Note that Cat 2 is able to achieve the optimum effect in Equation (7) if, for every $j(j=0,1,2)$, the condition $P_{j} \leq \frac{2}{3}$ is satisfied. Indeed, if $P_{0}>\frac{2}{3}$, then $P_{1}+P_{2}<\frac{1}{3}$ and $\omega_{0}<\frac{1}{3}$ (and similarly in the remaining cases). Let us now consider the situation of Cat 1 . This player moves first in each iteration of the game. Condition (6) can be written as follows:

$$
\begin{aligned}
& \left(1-L_{1}\right)\left(1-l_{0}\right) P_{0}+\left(1+L_{2}\right)\left(1+l_{0}\right) P_{0}+\left(1+L_{2}\right)\left(1-l_{1}\right) P_{1}+\left(1-L_{1}\right)\left(1+l_{2}\right) P_{2}=4 / 3, \\
& \left(1-L_{2}\right)\left(1+l_{0}\right) P_{0}+\left(1+L_{0}\right)\left(1+l_{1}\right) P_{1}+\left(1-L_{2}\right)\left(1-l_{1}\right) P_{1}+\left(1+L_{0}\right)\left(1-l_{2}\right) P_{2}=4 / 3, \\
& \left(1+L_{1}\right)\left(1-l_{0}\right) P_{0}+\left(1-L_{0}\right)\left(1+l_{1}\right) P_{1}+\left(1-L_{0}\right)\left(1-l_{2}\right) P_{2}+\left(1+L_{1}\right)\left(1+l_{2}\right) P_{2}=4 / 3,
\end{aligned}
$$

Let us suppose that there exist (if $P_{j} \leq \frac{2}{3}$ for any $j$ ) parameters $l_{j}$, such that Condition (7) is satisfied: Cat 2 achieves diet completeness.

Substituting Formula (9) for the probability $P_{j}$ into Formula (10) immediately gives:

$$
\begin{aligned}
& L_{1}=L_{2}, \\
& L_{0}=L_{2}, \\
& L_{0}=L_{1} .
\end{aligned}
$$


These conditions describe the set of Cat 1's optimal strategies. This player must use the strategy characterized by three (independent) equal conditional probabilities.

In the next section, we analyze the optimal strategies for both players in terms of their division into two types: intransitive and transitive.

\section{Optimal Intransitive and Transitive Strategies}

We say that a player prefers Food 1 to Food $0(1 \succ 0)$ when he/she is willing to choose Food 1 more often than Food 0 from the offered pair $(0,1)\left(P\left(C_{1} \mid B_{2}\right)>P\left(C_{0} \mid B_{2}\right)\right)$. The situation corresponds to an intransitive choice if one of the following two conditions is satisfied:

(1) $P\left(C_{0} \mid B_{2}\right)<P\left(C_{1} \mid B_{2}\right), P\left(C_{1} \mid B_{0}\right)<P\left(C_{2} \mid B_{0}\right), P\left(C_{2} \mid B_{1}\right)<P\left(C_{0} \mid B_{1}\right)$,

(2) $P\left(C_{0} \mid B_{2}\right)>P\left(C_{1} \mid B_{2}\right), P\left(C_{1} \mid B_{0}\right)>P\left(C_{2} \mid B_{0}\right), P\left(C_{2} \mid B_{1}\right)>P\left(C_{0} \mid B_{1}\right)$.

These two conditions can be written (by using the normalization of the probability measure to one) in the following form:

$$
\begin{aligned}
P\left(C_{0} \mid B_{2}\right) & <\frac{1}{2}, P\left(C_{1} \mid B_{0}\right)<\frac{1}{2}, P\left(C_{2} \mid B_{1}\right)<\frac{1}{2}, \\
P\left(C_{0} \mid B_{2}\right) & >\frac{1}{2}, P\left(C_{1} \mid B_{0}\right)>\frac{1}{2}, P\left(C_{2} \mid B_{1}\right)>\frac{1}{2} .
\end{aligned}
$$

Let us see how different types of strategies of individual players are achievable. The conditions under which Cat 2 makes his decision are similar to those considered in the work [10] (there, it was a game with nature). This player can select its optimal strategy only if $P_{j} \leq \frac{2}{3}$, for $j=0,1,2$. It turns out that under this assumption, this strategy can always be transitive, but not always intransitive. This can be illustrated graphically. Figure 3 presents the areas of frequency $\left(P_{0}, P_{1}, P_{2}\right)$ for which Cat 2's optimal strategies exist. It is the range (for 10,000 randomly selected points) of mapping (defined by Equations (9)) of the three-dimensional cube of parameters $t_{j}$ into a triangle $\left(P_{0}, P_{1}, P_{2}\right)$.

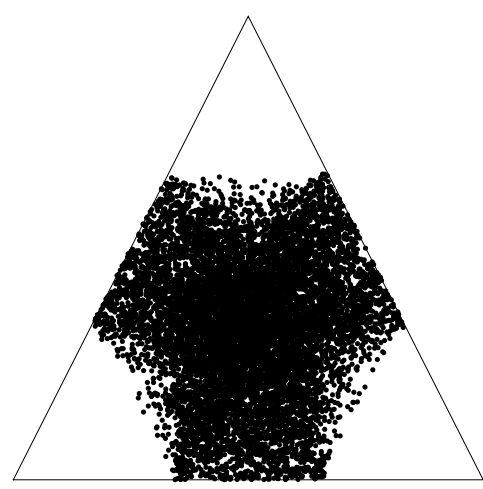

all

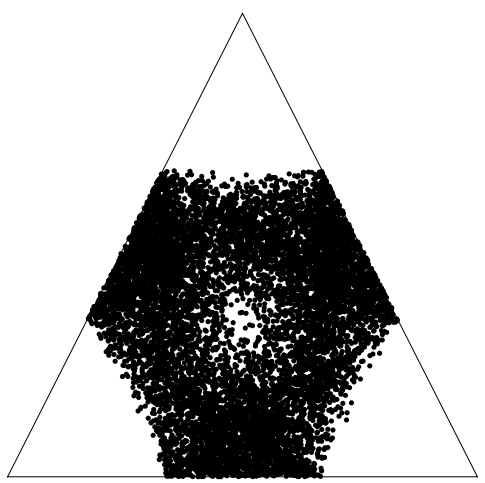

transitive

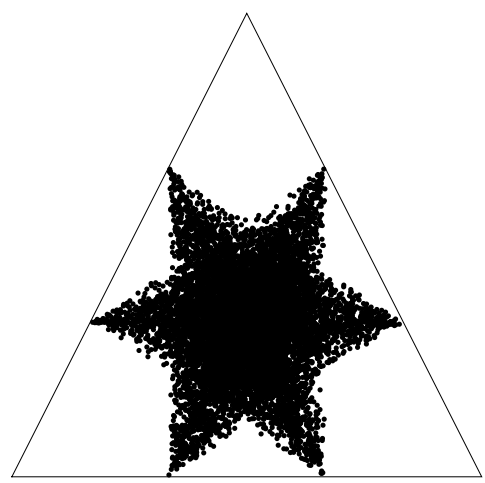

intransitive

Figure 3. Availability (for Cat 2) of different types of strategies.

Probabilities $\left(P_{0}, P_{1}, P_{2}\right)$ for which Cat 2 can identify the optimal intransitive strategy form a six-armed star composed of two triangles (any of them corresponding to one of two possible intransitive 
orders). Both the optimal strategies of any type and optimal transitive strategies correspond to a regular hexagon specified by conditions $P_{j} \leq \frac{2}{3}, j=0,1,2$.

The situation of the other player (Cat 1 ) is completely different. Cat 1 selects at the final stage of each iteration of the game. This movement reveals his preference for one food over another from the pair.

As pointed out above, Cat 1's optimal strategies (assuming that the second player reaches the optimal strategy) satisfy Condition (11). Therefore:

$$
Q\left(C_{0} \mid B_{2}\right)=Q\left(C_{1} \mid B_{0}\right)=Q\left(C_{2} \mid B_{1}\right)
$$

This means that either (12) or (13) is satisfied. This means that Cat 1 has to make intransitive choices in order to achieve the optimal result!

\section{Quantum Cats}

\subsection{One-Qubit Pure Strategies}

In this section, we use a different method for obtaining conditional probabilities (provided by the quantum games theory), which describe the preferences of the decision makers, Cat 1 and Cat 2, over pairs of food. This method was first used in the work [11] and is based on the concept of the so-called mutually unbiased bases:

Definition 2. Two orthonormal bases $\mathcal{A} \equiv\left\{\left|\psi_{0}\right\rangle \ldots\left|\psi_{N-1}\right\rangle\right\}$ and $\mathcal{B} \equiv\left\{\left|\varphi_{0}\right\rangle \ldots\left|\varphi_{N-1}\right\rangle\right\}$ in Hilbert space $\mathbb{C}^{N}$ are mutually unbiased if:

$$
\left|\left\langle\psi_{i} \mid \varphi_{j}\right\rangle\right|^{2}=\frac{1}{N}
$$

for any $0 \leq i, j \leq N-1$.

For a two-dimensional Hilbert space, three mutually unbiased bases are given as follows:

$$
\begin{aligned}
& \{|0\rangle,|1\rangle\}, \\
& \left\{\frac{|0\rangle+|1\rangle}{\sqrt{2}}, \frac{|0\rangle-|1\rangle}{\sqrt{2}}\right\}, \\
& \left\{\frac{|0\rangle+i|1\rangle}{\sqrt{2}}, \frac{|0\rangle-i|1\rangle}{\sqrt{2}}\right\} .
\end{aligned}
$$

This set is very important for our considerations. It is worth mentioning here that mutually unbiased bases led Wiesner [41] to begin research into quantum cryptography, before asymmetric key cryptography was invented!These bases play also an important role in the universality of quantum market games [42-44].

Let us turn to the construction of conditional probabilities that define the strategies of the players. Let us denote three different, mutually unbiased bases of two-dimensional Hilbert space as:

$$
\left\{|1\rangle_{0},|2\rangle_{0}\right\},\left\{|0\rangle_{1},|2\rangle_{1}\right\},\left\{|0\rangle_{2},|1\rangle_{2}\right\}=\left\{(1,0)^{T},(0,1)^{T}\right\}
$$

The strategy of choosing food $k$ when the offered food pair does not contain food 1 is denoted by $|k\rangle_{l}$ $(k, l=0,1,2$ and $k \neq l)$. 
A family $\{|z\rangle\}(z \in \overline{\mathbb{C}})$ of vectors:

$$
|z\rangle:=|0\rangle_{2}+z|1\rangle_{2}=|0\rangle_{1}+\frac{1-z}{1+z}|2\rangle_{1}=|1\rangle_{0}+\frac{1+i z}{1-i z}|2\rangle_{0},
$$

defined by the parameters of the heterogeneous coordinates of the projective space $\mathbb{C} P^{1}$ represents all strategies spanned by the base vectors.

The coordinates of the same strategy $|z\rangle$ measured in three different bases (15) define the players' preferences toward a food pair represented by the base vectors. The squares of their moduli are measured after normalization of the conditional probability of cats making a decision in choosing a particular product, when the choice is related to the suggested food pair. A diagram of the quantum variant of the game is shown in Figure 4. It is similar to the previous one in Figure 2, since the difference is hidden in the process of implementing the game only.

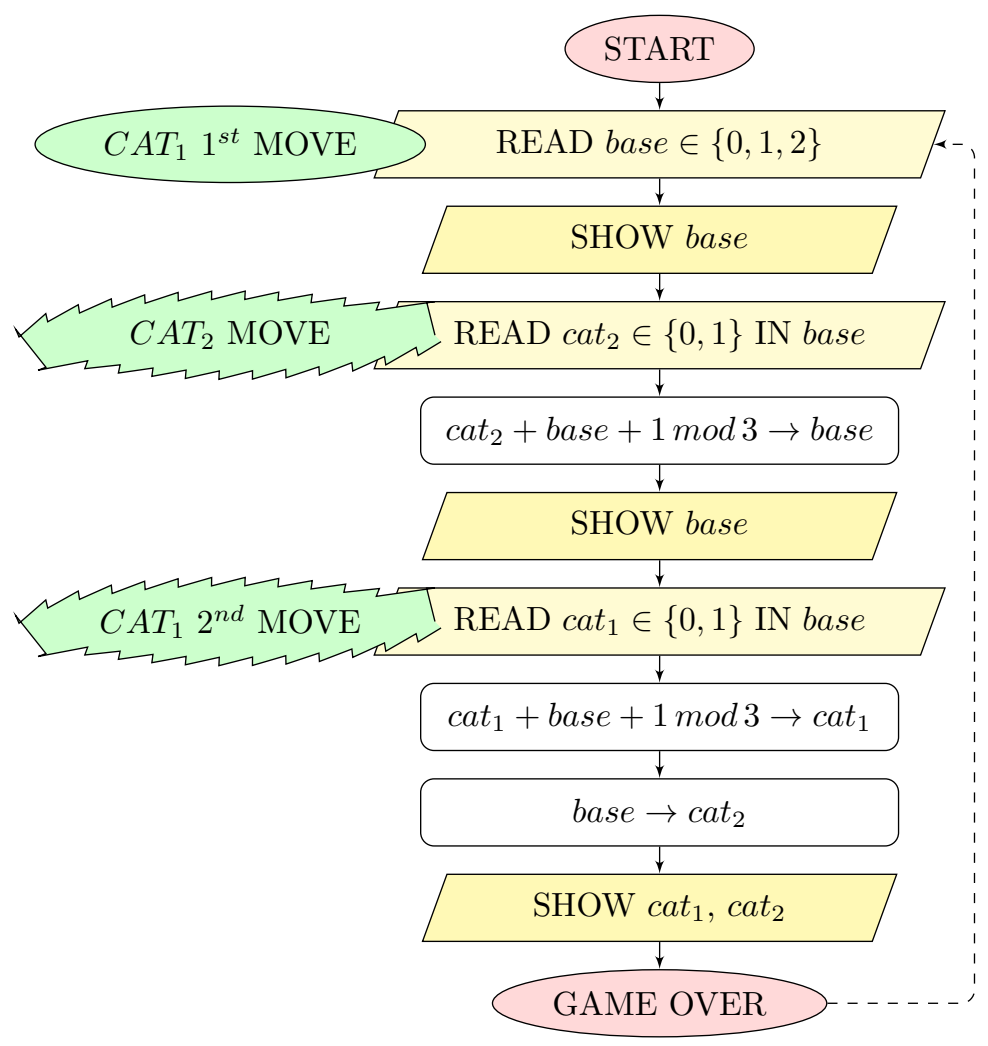

Figure 4. Flow diagram of each iteration of the quantum variant of the game.

The player (Cat 1 or Cat 2 in our game) makes a decision to choose the preferred food from a pair with the following probabilities:

$$
\begin{array}{ll}
P\left(C_{0} \mid B_{2}\right)=\frac{1}{1+|z|^{2}}, & P\left(C_{1} \mid B_{2}\right)=\frac{|z|^{2}}{1+|z|^{2}}, \\
P\left(C_{0} \mid B_{1}\right)=\frac{1}{1+\left|\frac{1-z}{1+z}\right|^{2}}, & P\left(C_{2} \mid B_{1}\right)=\frac{\left|\frac{1-z}{1+z}\right|^{2}}{1+\left|\frac{1-z}{1+z}\right|^{2}}, \\
P\left(C_{1} \mid B_{0}\right)=\frac{1}{1+\left|\frac{1+i z}{1-i z}\right|^{2}}, & P\left(C_{2} \mid B_{0}\right)=\frac{\left|\frac{1+i z}{1-i z}\right|^{2}}{1+\left|\frac{1+i z}{1-i z}\right|^{2}} .
\end{array}
$$


It is convenient to parametrize $|z\rangle$ by points of the sphere $S_{2} \simeq \overline{\mathbb{C}}$ by using the stereographic projection, which establishes bijection between elements of $\overline{\mathbb{C}}$ and the points of $S_{2}$.

The conditional probabilities can now be written in the following form:

$$
\begin{array}{ll}
P\left(C_{0} \mid B_{2}\right)=\frac{1-x_{3}}{2}, & P\left(C_{1} \mid B_{2}\right)=\frac{1+x_{3}}{2}, \\
P\left(C_{0} \mid B_{1}\right)=\frac{1+x_{1}}{2}, & P\left(C_{2} \mid B_{1}\right)=\frac{1-x_{1}}{2}, \\
P\left(C_{1} \mid B_{0}\right)=\frac{1+x_{2}}{2}, & P\left(C_{2} \mid B_{0}\right)=\frac{1-x_{2}}{2} .
\end{array}
$$

Note that the probabilities (17) are parametrized analogously to the classical model. In this case, however, we have sphere points, so the condition $x_{1}^{2}+x_{2}^{2}+x_{3}^{2}=1$ must be satisfied.

A careful reader certainly noticed that we abstained from introducing too much of the game theoretical terminology. The problem can be easily reformulated in the language of quantum game theory $[7,8]$. Another interesting approach would be to adopt the framework developed by Lambert-Mogiliansky and Martinez-Martinez [45]; $c f$. also [46]. This framework allows one to incorporate the type indeterminacy of agents $[45,47,48]$. In our model, the agents are of definite type.

\subsection{Availability of Different Types of One-Qubit Strategies}

The availability of various types of Cat 2's one-qubit optimal strategies is illustrated in Figure 5 (see [11]).

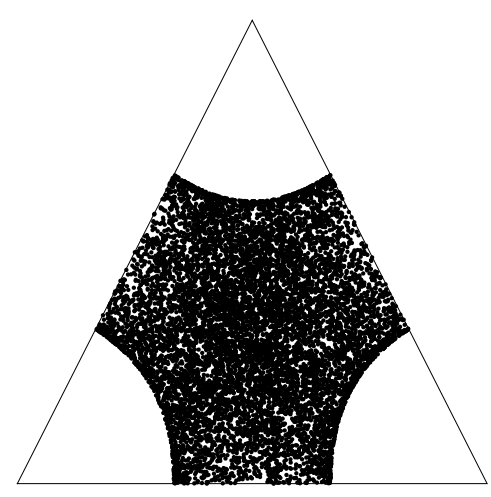

all

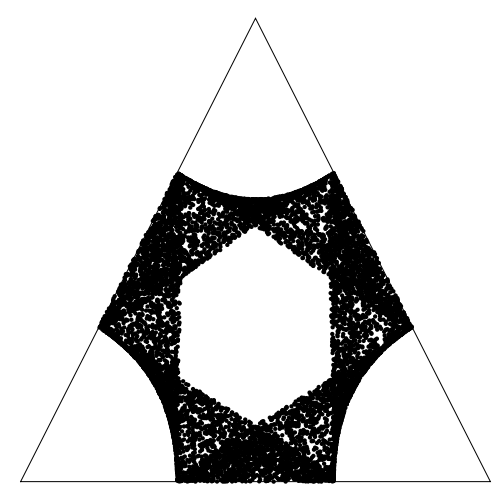

transitive

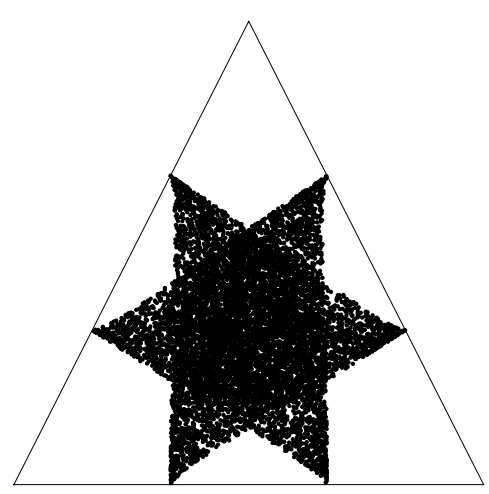

intransitive

Figure 5. Availability (for Cat 2) of different types of one-qubit strategies.

Here, we see a fundamental contrast between the quantum and classical model. First of all, the area corresponding to the optimal transitive strategies does not cover the entire area of the optimal strategy of any type (transitive or intransitive). Transitive optimal strategies do not appear within the boundaries of the hexagon-like figure in the central part of the triangle.

This means that for some frequencies $\left(P_{0}, P_{1}, P_{2}\right)$, Cat 2 is able to achieve optimal results only due to the intransitive strategy!Detailed quantitative analysis of the second player's optimal strategies (Figures 3 and 5) is analogous to that considered in [11]. Readers interested in this issue should refer to this paper. 
Let us analyze the Cat 1 situation in the case of a one-qubit pure strategy, at the final stage of each iteration of the game. The probabilities $Q\left(C_{k} \mid B_{j}\right)$ are in the form (17). In order to distinguish this from the strategy of the other player, we will use $X_{l}$ instead of $x_{l}$.

Assuming that Cat 2 achieves the optimal strategy, the optimality conditions (8) for Cat 1 will simplify to:

$$
\begin{aligned}
& X_{1}-X_{3}=0, \\
& X_{2}+X_{3}=0, \\
& X_{1}+X_{2}=0 .
\end{aligned}
$$

Since $X_{1}^{2}+X_{2}^{2}+X_{3}^{2}=1$, we obtain two optimal strategies that are characterized by two points $\left(X_{1}, X_{2}, X_{3}\right)$ of a two-dimensional sphere:

$$
\begin{gathered}
X_{1}=-\frac{1}{\sqrt{3}}, X_{2}=\frac{1}{\sqrt{3}}, X_{3}=-\frac{1}{\sqrt{3}}, \\
X_{1}=\frac{1}{\sqrt{3}}, X_{2}=-\frac{1}{\sqrt{3}}, X_{3}=\frac{1}{\sqrt{3}} .
\end{gathered}
$$

Both of these strategies are intransitive.

It is worth noting that in the language of quantum game theory, one-qubit strategies are pure strategies, of equal informative values (zero entropy). Hence, treating them in an equivalent way is natural and does not raise any controversy (Laplace's principle of insufficient reason) in the classical model. In this case, we used mixed strategies, which may provide different pieces of information. Pure strategies that we can identify with eight choice functions:

$$
f_{k}:\{(0,1),(0,2),(1,2)\} \rightarrow\{0,1,2\}, \quad k=0, \ldots, 7,
$$

do not lead to optimal strategies.

\section{Discussion}

The variant of the game considered in this paper allows one to explore the availability of different types of optimal strategies of both players (as opposed to earlier models, which were related to the situation of one player only). They make their decisions in a variety of contexts (depending on the decision made by the other player), which affects their possibility to use optimal intransitive and transitive strategies. The most interesting variant of the game has been analyzed, i.e., the variant where the circumstances provide both players with the opportunity to achieve their optimal strategies.

It appears that the only optimal strategies of Cat 1 (second move) are intransitive strategies. This is indeed an interesting result. This player performs two moves in the game. In the first one, he chooses (with frequency $P_{j}$ ) one of the foods. The food is included in the pair from which he makes the final selection. Therefore, he has a partial influence on the composition of this pair (Cat 2 does not have such a possibility). It might seem that such a possibility should help to him to achieve an optimal strategy in the sense that he has more freedom in choosing the type of strategy. It is quite opposite, however: he can only use an intransitive strategy. The other player (Cat 2) in the classical variant of the game can always use a transitive strategy (in certain specific conditions, also an intransitive strategy; see Figure 3). 
Replacing classical strategies of the players with one-qubit quantum strategies will increase the importance of intransitive strategies. In order to achieve an optimal effect, both players must apply intransitive strategies at certain $\left(P_{0}, P_{1}, P_{2}\right)$ frequencies!Moreover, the one-qubit strategies are pure strategies; they have an equivalent mathematical and information status (in contrast with the mixed strategies in the classical model).

\section{Conclusions}

The model discussed in the present paper, despite its mathematical simplicity, clearly illustrates how the type of preferences depends on the context of the decision to be made and on the behavior of the other players. The rejection of intransitive strategies as undesirable strategies is an unnecessary limitation. A distinction between intransitive and transitive strategies does not necessarily mean a distinction between irrational and rational strategies. However, it may well describe the decision-making process from an entirely different point of view: the context of choice (e.g., whether and how the freedom of choice with regard to the decisions we make is affected by the adopted rules or the behavior of other players).

It is worth mentioning that at present, we can observe a rapidly growing literature where the ideas of quantum mechanics are proposed to explain problems of behavioral and social sciences [49-54]. The formalism of quantum mechanics can be useful in providing an explanation of the violations of transitivity in the decision-making process (see [46]). We are still not fully aware of the implications of quantum theory in computer science models, machine learning and especially in decision-making. The work that has been done in this field indicates significant progress in the development of this part of our knowledge.

\section{Acknowledgments}

This work was supported by the Polish National Science Centre under Project Number DEC-2011/01/B/ST6/07197.

\section{Author Contributions}

Marcin Makowski, Edward W. Piotrowski and Jan Sładkowski conceived of the problem, designed the simulations, analyzed the data, and contributed to the writing of the manuscript. All authors have read and approved the final manuscript.

\section{Conflicts of Interest}

The authors declare no conflict of interest.

\section{References}

1. Von Neumann, J.; Morgenstern, O. Theory of Games and Economic Behavior; Princeton University Press: Princeton, MA, USA, 1944.

2. Straffin, P.D. Game Theory and Strategy; The Mathematical Association of America: Washington DC, USA, 1992. 
3. Rasmusen, E. Games and Information: An Introduction to Game Theory; Wiley-Blackwell: Malden, MA, USA, 2007.

4. Nielsen, M.A,; Chuang, I.L. Quantum Computation and Quantum information; Cambridge University Press: New York, NY, USA, 2000.

5. Eisert, J.; Wilkens, M.; Lewenstein, M. Quantum games and quantum strategies. Phys. Rev. Lett. 1999, 83, 3077-3080.

6. Meyer, D. Quantum strategies. Phys. Rev. Lett. 1999, 8, 1052-1055.

7. Flitney, A.P.; Abbott, D. An introduction to quantum game theory. Fluct. Noise Lett. 2002, 2, R175-R187.

8. Piotrowski, E.W.; Sładkowski, J. An invitation to quantum game theory. Int. J. Theor. Phys. 2003, 42, 1089-1099.

9. Piotrowski, E.W.; Sładkowski, J. The next stage: Quantum game theory. In Mathematical Physics Research at the Cutting Edge; Benton, C.V., Ed.; Nova Science Publishers: New York, NY, USA, 2004; pp. 247-268.

10. Piotrowski, E.W.; Makowski, M. Cat's dilemma-transitivity vs. intransitivity. Fluct. Noise Lett. 2005, 5, L85-L96.

11. Makowski, M.; Piotrowski, E.W. Quantum cat's dilemma: An example of intransitivity in a quantum game. Phys. Lett. A 2006, 355, 250-254.

12. Makowski, M. Transitivity vs. intransitivity in decision making process-an example in quantum game theory. Phys. Lett. A 2009, 373, 2125-2130.

13. Makowski, M.; Piotrowski, E.W. Transitivity of an entangled choice. J. Phys. A Math. Theor. 2011, 44, 075301.

14. Makowski, M.; Piotrowski, E.W. Decisions in elections-transitive or intransitive quantum preferences. J. Phys. A Math. Theor. 2011, 44, 215303.

15. Makowski, M.; Piotrowski, E.W. When I cut, you choose method implies intransitivity. Physica A 2014, 415, 189-193.

16. Steinhaus, H. Memoires and Notes; Aneks: London, UK, 1992. (in Polish)

17. Anand, P.; Pattanaik, P.K.; Puppe, C. Introduction. In Handbook of Rational and Social Choice; Anand, P., Pattanaik, P.K., Puppe, C., Eds.; Oxford University Press: New York, NY, USA, 2009; pp.1-20.

18. Von Wright, G.H. The Logic of Preference: An Essay; Edinburgh University Press: Edinburgh, UK, 1963.

19. Grosenick, L.; Clement, T.S.; Fernald, R.D. Fish can infer social rank by observation alone. Nature 2007 445, 429-432.

20. Tullock, G. The irrationality of intransitivity. Oxf. Econ. Pap. 1964, 16, 401-406.

21. Fishburn, P.C. Nontransitive Preferences in Decision Theory. J. Risk Uncertain. 1991 4, 113-134.

22. Fishburn, P.C. Nontransitive measurable utility. J. Math. Psychol. 1982, 26, 31-67.

23. Arrow, K.J. Social Choice and Individual Values; Yale University Press: New York, NY, USA, 1951.

24. Poddiakov, A.N. Intransitive Character of Superiority Relations and Decision-making. Psychology 2006, 3, 88-111. 
25. Tversky, A. Intransitivity of preferences. Psychol. Rev. 1969, 76, 31-48.

26. Gardner, M. Mathematical Games: The Paradox of the Nontransitive Dice and the Elusive Principle of Indifference. Sci. Am. 1970, 223, 110-114.

27. Penney, W. Problem 95: Penney-ante. J. Rec. Math. 1969, 241-258.

28. Boddy, L. Interspecific combative interactions between wood-decaying basidiomycetes. FEMS Microbiol. Ecol. 2000, 31, 185-194.

29. Sinervo, B.; Lively, C.M. The rock-paper-scissors game and the evolution of alternative male strategies. Nature 1996, 380, 240-243.

30. Shafir, S. Intransitivity of preferences in honey bees: Support for'comparative'evaluation of foraging options. Anim. Behav. 1994, 48, 55-67.

31. Pahikkala, T.; Waegeman, W.; Tsivtsivadze, E.; Salakoski, T.; De Baets, B. Learning intransitive reciprocal relations with kernel methods. Eur. J. Oper. Res. 2010, 206, 676-685.

32. Klimenko, A.Y. Abstract competition and competitive thermodynamics. Phil. Trans. R. Soc. A 2013, 371, 1-16.

33. Halpern, J.Y. Intransitivity and vagueness. Rev. Symbol. Log. 2008, 1, 530-547.

34. Tversky, A.; Kahneman, D. Choices, Values, and Frames; Cambridge University Press: New York, NY, USA, 2000.

35. Karni, E. Decision Making Under Uncertainty: The Case of State-Dependent Preference; Harvard University Press: Cambridge, UK, 1985.

36. Tversky, A.; Simonson, I. Context-dependent preferences. Manag. Sci. 1993, 39, 1179-1189.

37. Aerts, D.; Sozzo, S.; Veloz, T. Quantum Structure in Cognition and the Foundations of Human Reasoning. 2014, arXiv:1412.8704v1 [cs.AI], http://arxiv.org/pdf/1412.8704.pdf.

38. Khrennikova, P.; Haven, E.; Khrennikov, A. An application of the theory of open quantum systems to model the dynamics of party governance in the US Political System. Int. J. Theor. Phys. 2013, 53, 1346-1360.

39. Brams, S.J.; Taylor, A.D. Fair Division-From Cake-cutting to Dispute Resolution; Cambridge University Press: New York, NY, USA, 1996.

40. Steinhaus, H. Mathematical Snapshots; Oxford University Press: New York, NY, USA, 1996.

41. Wiesner, S. Conjugate coding. Sigact News 1983, 15, 78-88.

42. Piotrowski, E.W.; Sładkowski, J. Quantum Market Games. Physica A 2002, 312, 208-216.

43. Piotrowski, E.W.; Sładkowski, J. Quantum-like Approach to Financial Risk: Quantum Anthropic Principle. Acta Phys. Pol. B 2001, 32, 3873-3879.

44. Pakuła, I.; Piotrowski, E.W.; Sładkowski, J. Universality of measurements on quantum markets. Physica A 2007, 385, 397-405.

45. Lambert-Mogiliansky, A.; Martinez-Martinez, I. Basic Framework for Games with Quantum-like Players. PSE Working Pap. 2014, 2014, 1-21.

46. Lambert-Mogiliansky, A.; Zamir, S.; Zwirn, H. Type indeterminacy: A model of the KT(Kahneman-Tversky)-man. J. Math. Psychol. 2009, 53, 349-361.

47. Kvam, P.D.; Busemeyer, J.R.; Lambert-Mogiliansky, A. An Empirical Test of Type-Indeterminacy in the Prisoner's Dilemma. In Quantum Interaction; Atmanspacher, H., Haven, E., Kitto, K., Raine, D., Eds; Springer: Berlin/Heidelberg, Germany, 2014; pp. 213-224. 
48. Lambert-Mogiliansky, A.; Busemeyer, J.R. Quantum type indeterminacy in dynamic decision-making: Self-control through identity management. Games 2012, 3, 97-118.

49. Brandenburger, A.; La Mura, P. Team Decision Problems with Classical and Quantum Signals. 2015, arXiv:1107.0237v3 [quant-ph].

50. Busemeyer, J.R.; Wang, Z.; Townsend, J.T. Quantum dynamics of human decision making. J. Math. Psychol. 2006, 50, 220-241.

51. Brandenburger, A. The relationship between quantum and classical correlation in games. Games Econ. Behav. 2010, 69, 175-183.

52. La Mura, P. A Double-Slit Experiment for Non-Classical Interference Effects in Decision Making. Top. Cogn. Sci. 2014, 6, 58-62.

53. Yukalov, V.; Sornette, D. Conditions for Quantum Interference in Cognitive Sciences. Top. Cogn. Sci. 2014, 6, 79-90.

54. Busemeyer, J.R.; Bruza, P.D. Quantum Models of Cognition and Decision; Cambridge University Press: New York, NY, USA, 2012.

(c) 2015 by the authors; licensee MDPI, Basel, Switzerland. This article is an open access article distributed under the terms and conditions of the Creative Commons Attribution license (http://creativecommons.org/licenses/by/4.0/). 